\title{
On the Modelling of Weights of Kids to Enhance Growth in a Local Goat Population Under Tunisian Arid Conditions. The Maternal Effects.
}

ATOUI AHLEM ( $\sim$ ahlematoui@gmail.com )

Institut des Regions Arides

Najari Sghaier

Institut des Regions Arides de Medenine: Institut des Regions Arides

Clara Díaz2 Clara Díaz

INIA MADRID

mouldi Abdennebi

Institut des Regions Arides de Medenine: Institut des Regions Arides

Maria jesus carabano

INIA

Research Article

Keywords: local goat, maternal heritability, direct heritability, EBV, correlation, arid environment

Posted Date: November 9th, 2021

DOI: https://doi.org/10.21203/rs.3.rs-962125/v1

License: (c) (i) This work is licensed under a Creative Commons Attribution 4.0 International License.

Read Full License 
1 On the modelling of weights of kids to enhance growth in a local goat population under Tunisian arid conditions. The maternal effects.

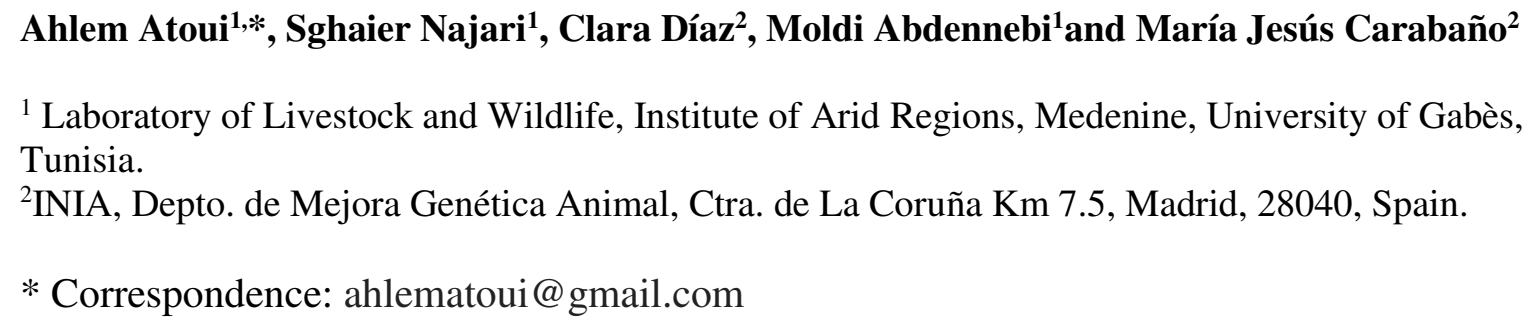

Abstract : To provide the local goat population with an adequate genetic management scheme under arid environment, the study aimed to evaluate the use of fourteen alternative models attempting to evaluate to dissection of additive genetic (AG) and permanent environmental (PE) components of direct and maternal effects and their consequences on estimated breeding values. Records of 938 local kids over a period of 16 years were used in this study. Data were split into four groups corresponding to four periods along the weight recording period. Periods 1/2/3/4 contained weights in an interval with upper-lower limits of 1-20/25-60/65-120/125-150 days of age. Models including or ignoring maternal genetic or permanent environmental effects were fitted for all traits. For all periods, the best models were those including the AG component for both direct and maternal effects and the direct PE effect. Heritability estimates of both the direct and maternal effects ranged from low $(0.02$ for maternal heritability in P1) to moderate ( 0.17 for direct and maternal heritability in P2 and P3). Period 1 showed the lowest values for heritability of both direct and maternal effects, which also showed the largest estimate of the ratio of residual to total variance (around 0.2) compared with the other periods, withdecreasing ratios as age increased (from 0.13 for P2 to 0.07 for P4). Both direct and maternal EBVs showed high correlations between models fitting direct AG and PE effects. For direct EBVs, correlations were above 0.99, indicating that the same animals are expected to be selected under any model that includes those components, regardless of the maternal effects included. For maternal EBVs, correlations were also high, but slightly lower than for the DEBVs between models including DGP effects and maternal genetic effects. Overall, our recommendation for genetic evaluations of direct and maternal effectsin this population raised in extensive and harsh conditions is to use weight records preferably collected during the period of high milk production of dams, for which direct and maternal effects are expected to show full expression. Complete pedigrees and several generations of dam-progeny recording are needed to obtain a proper separation of environmental and genetic components. 
Keywords: local goat; maternal heritability; direct heritability; EBV; correlation; arid environment.

\section{Introduction}

Goat meat production is a significant source of income of farmers in the southern regions of Tunisia. Local breeds are often used because of their adaptation to the harsh conditions, but they are characterized by a slow growthrate what evenin a low-input system, it be self-sustainable becauseit does not provide enough margin to make a way of leaving for producers. In this context, developing a global strategy for the sector in this arid environment, to improve the pastoral farming system, as well as also, theproductive potentials of individuals without damaging the adaptive capacity is then,of great interest in this region.

Genetic improvement of growth ability of local breeds is a tool toenhance the economic sustainability of goat farming. To do so, recording of appropriate phenotypes (weights at certain ages) and pedigrees are needed to achieve this goal (Atoui et al. 2018; Barazandeh 2006). As in most extensive systems of production, improvement ofthe maternal ability is particularly relevant because young animals spend a long period with their dams being highly dependent on them. This maternal influence starts during gestation and in some cases may bemaintained during life conditioning the future productive performance of progeny through epigenetic effects. Genetic improvement programs are based on exploitation of genetic variation, identification of superior animals for specific traits or trait combinations, and widespread utilization of these animals in a population. In this context, knowledge of the maternal influence on pre and post weaning weights and of the correlation between these effects is fundamental for achieving unbiased estimates of the breeding values.

The total phenotypic variance for weight and growth traits in young animals is often divided into components of direct, maternal and residual effects. Because of the importance of a correct modelling of environmental and genetic effects to obtain unbiased estimates of breeding values, many previous studies have dealt with the estimation of (co)variance components for growth traits of young farm animals using different models that include direct and maternal components (Bahrein et al. 2007; Bijma 2006; Ghafouri et al. 2008; Mandal et al. 2006; Mavrogens et al. 1984; Mugambi et al. 2007). Disentangling the direct and maternal additive genetic components from each other and from other environmental effects on growth to yield unbiased estimates of breeding values is challenging, as pointed out by several authors (Bijma 2006; David et al. 2015; Meyer 1992b; Varona et al. 2015), but no clear 
solution is available for practical genetic evaluations. In fact, estimates of genetic parameters change widely across populations due to differences in statistical models (Meyer 1992b; Boujenane et al. 2002; Meyer 1992a), data structure in terms of information on dams and maternal ancestors (Meyer 1992b, Heydarpour et al. 2008; Maniatis et al. 2003) or breeds/species, because of differences in type of information per individual and type of production system (intensive in species as pigs or rabbits vs. extensive in small ruminants) (David et al. 2015).

Although from a biological point of view, inclusion of genetic and permanent environmental effects linked to both the animal producing the data and its dam seems to be most plausible, careful evaluation of alternative models considering the extent to which removing a particular source of environmental variance from a model affects other estimates, can provide useful information to interpret the sources of variation in the available phenotypes (Fischer et al. 2004; Gunia et al. 2011; Krejčová et al. 2008) as well as the bias generated in the estimates (Meyer 1992b) and on the impact of model on the breeding value estimation to predict direct genetic as well as the maternal genetic component.

In a previous study, a random regression and multiple trait models using data from the experimental station used in this study, collection of data at least in two periods, early and late stages of the kids rearing period on farm was recommended to provide reliable information on both direct and maternal effects. However, large discrepancies on estimates of genetic parameters from different models in those periods were observed. Moreover, the fact that information from many weights recorded in each animal were used in the analysis of the whole period from birth to 150 days of age, is not a realistic situation in the field.

In this context, the aim of this study was to evaluate the use of alternative models attempting to separate direct genetic, maternal genetic and maternal permanent environmental effects using data from separate periods where maternal influence is expected to change and, mimicking a more realistic situation, where only one or two weights could be recorded. A number of studies have dealt with these models in the past, but the consequences on estimated breeding values and possible implications for selection has been given little attention.

\section{Material and Methods}

\section{Animals and management}

All experimental goats were raised in the Arid Areas Institute of Médenine (IRA), in the South-East of Tunisia with an arid continental Mediterranean climate, with irregular and sporadic rains. The local goat population is a very heterogeneous group of animals from a 
genetic and morphological point of view. A photograph of the Tunisian goat is provided in figure 1. It is known for its small size, the average height is around $73 \mathrm{~cm}$ for males and only $60 \mathrm{~cm}$ for females. As for the average adult weight, it is $35 \mathrm{~kg}$ for males and $24 \mathrm{~kg}$ for females (Atoui et al. 2019; Najari 2005). The reproductive management of the flock is planned to obtain one kidding per year. The main mating period is from June to August. The season of kidding begins in October and continues until February, with a concentration during November and December. The suckling period lasts for 120 days on average. The female kids are mated for the first time between 12 and 18 months of age, depending on their season of birth. The number of goats mated per sire varied from 5 to 17 . Goats were randomly assigned to bucks. Bucks were replaced every 5 years with replacements coming from outside flocks in order to control inbreeding or from the experimental flock. Animals grazed in natural pastures during the day. In general, grazing pasture grasses covered about $70 \%$ of breeding animals feed requirements. The remaining 30\% was covered by a supplementation provided during the mating (600 g/day), the last month of pregnancy and the beginning of lactation (750 g/day). All animals were provided with water allowed twice a day, before and after grazing (Atoui et al. 2018; Atoui et al. 2019).

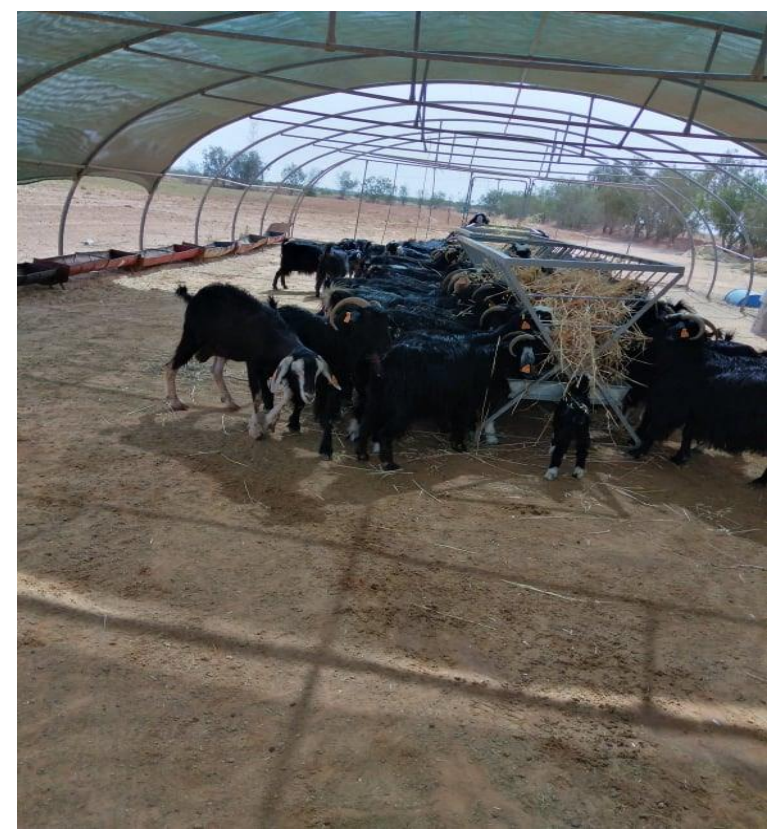

Fig.1 Photograph of the Tunisian local goat population.

The data used in the present study were collected between 1998 and 2014 from a total of 938 kids. Since the start of the kidding period and till 150 days of age, kids were weighed once 
every two or three weeks. The available data were divided into four data sets corresponding to four age periods 1-20 (P1), 25-60 (P2), 65-120 (P3) and 125-150 (P4) days. Periods were defined to identify different stages in the kid-dam feeding. Period 1 represents the early period after birth, P2 coincides with the period of increasing milk production of the dam, P3 is the after production peak up to weaning period and $\mathrm{P} 4$ is the period after weaning.

From previous studies (Atoui et al. 2019), the effects of year and month of birth, age and weight of the dam at birth, sex and type of birth (single, double, etc.) and age of the kid at weighing were found to be significantly affecting the weights of kids when weights from all ages were analysed jointly. In order to confirm the significance of those effects for each of the periods under study, separate ANOVA analyses for each period were carried out.

For records belonging to each of the periods, the full model contained both direct and maternal effects including the genetic and permanent environmental components of each effect. The general equation of this model was:

$$
\mathrm{y}_{\mathrm{ijklndo}}=\mathrm{FE}_{\mathrm{ijk}}+\mathrm{a}_{\mathrm{n}}^{\mathrm{d}}+\mathrm{p}_{\mathrm{n}}^{\mathrm{d}}+\mathrm{a}_{\mathrm{d}}^{\mathrm{m}}+\mathrm{p}_{\mathrm{d}}^{\mathrm{m}}+\varepsilon_{\mathrm{ijklndo}}
$$

, where, $\mathrm{y}_{\mathrm{ijkndo}}$ is the record from $\mathrm{n}^{\text {th }}$ animal within each period, $\mathrm{FE}_{\mathrm{ijk}}$ are fixed environmental effects fitted for all models, $a_{n}^{d}, p_{n}^{d}, a_{d}^{m}, p_{d}^{m}$ are the random direct additive genetic (DAG), and permanent environmental (DPE) effects for animal $n$ and maternal additive genetic (MAG) and permanent environmental (MPE) components for its dam d, respectively, and, $\varepsilon_{\mathrm{ijkndo}}$ is the residual effect.

$$
\text { In [1], } \mathrm{FE}_{\mathrm{ijkl}}=\mathrm{YM}_{\mathrm{i}}+\mathrm{AD}_{\mathrm{j}}+\mathrm{WD}_{\mathrm{k}}+\mathrm{ST}_{\mathrm{l}}+\sum_{\mathrm{r}=1}^{2} \beta_{\mathrm{rl}} \varphi_{\mathrm{r}}\left(\mathrm{t}_{\mathrm{ij}}\right) \text {, }
$$

where $\mathrm{YM}_{\mathrm{i}}$ is the year×month effect ( $\left.\mathrm{i}=1,13\right), \mathrm{AD}_{\mathrm{j}}$ is the age of dam at kidding effect $(j=1 \ldots \ldots 13), W_{k}$ is the weight of dam at kidding effect $(k=1, \ldots 3), S_{1}$ is the sex $\times$ type of birth effect $(1=1, \ldots 6), \beta_{\mathrm{rl}}$ are the fixed regression coefficients nested to the sex $\times$ type of birth combination.

The associated variance-covariance structure for random effects in [1] was:

$\mathrm{V}(\mathbf{a})=\left[\begin{array}{cc}\sigma_{\mathrm{ad}}^{2} & \sigma_{\mathrm{adm}} \\ \sigma_{\mathrm{adm}} & \sigma_{\mathrm{am}}^{2}\end{array}\right] \otimes \mathbf{A} ; \mathrm{V}(\mathbf{p})=\left[\begin{array}{cc}\sigma_{\mathrm{pd}}^{2} \mathbf{I}_{\mathrm{pd}} & 0 \\ 0 & \sigma_{\mathrm{pm}}^{2} \mathbf{I}_{\mathrm{pm}}\end{array}\right] ; \mathrm{V}(\mathbf{e})=\sigma_{\varepsilon}^{2} \mathbf{I}_{\mathrm{e}}$

, where vectors $\mathbf{a} / \mathbf{p}$ contain the additive genetic/permanent environmental direct and maternal effects, $\sigma_{\mathrm{ad}}^{2}$ and $\sigma_{\mathrm{am}}^{2}$ are the variances for DAG and MAG components, respectively, $\sigma_{\mathrm{adm}}$ is the covariance between those components, $\mathbf{A}$ is the numerator relationship matrix, $\sigma_{\mathrm{pd}}^{2}$ and 
$\sigma_{\mathrm{pm}}^{2}$ are the variances for DPE and MPE components, respectively, $\mathbf{I}_{\mathrm{pd}}$ is a diagonal matrix of order equal to the number of kids with records, $\mathbf{I}_{\mathrm{pm}}$ is a diagonal matrix of order equal to the number of dams of kids with records, $\sigma_{\varepsilon}^{2}$ is the residual variance and $\mathbf{I}_{\mathrm{e}}$ is a diagonal matrix of order equal to the number of observations. For models where the MPE effect is fitted, we are accounting for a mixture of within and between kidding effects.

In addition to the full model, a series of 14 reduced single-trait linear animal models that differed in the (co)variance components fitted were considered. The models expanded from a simple animal model for either the animal or dam effects to the comprehensive direct and maternal effects model in [1]. The idea behind this exercise was to determine the relative relevance of direct vs. maternal effects depending on the available information of weights along age (periods P1 to P4). The mixed models used in each period are summarized in terms of the variance components fitted in Table 1. Apart from model number, coding for models in a 1/0mode is provided to indicate presence or absence of DAG, DPE, MAG and MPE effects and covariance between DAG and MAG effects in the model, following this order. For example, model 10101 would be a model including DAG, MAG and covariance between them and excluding the DPE and MPE components.

Table 1 (Co) variance components for direct and maternal effects fitted in the models used in the analysis of each period.

\begin{tabular}{|c|c|c|c|c|c|c|}
\hline $\mathbf{N}$ & Code $^{1}$ & $\overline{\sigma_{\text {ad }}^{2}}$ & $\sigma_{p d}^{2}$ & $\sigma_{\mathrm{adm}}$ & $\overline{\sigma_{a m}^{2}}$ & $\sigma_{\mathrm{pm}}^{2}$ \\
\hline 1 & 11111 & $\checkmark$ & $\checkmark$ & & v & $\checkmark$ \\
\hline 2 & 11110 & $\checkmark$ & $\checkmark$ & & $\checkmark$ & $\checkmark$ \\
\hline 3 & 11101 & $\checkmark$ & $\checkmark$ & & $\checkmark$ & \\
\hline 4 & 11100 & $\checkmark$ & $\checkmark$ & & $\checkmark$ & \\
\hline 5 & 11010 & $\checkmark$ & $\checkmark$ & & & $\checkmark$ \\
\hline 6 & $1100_{-}$ & $\checkmark$ & $\checkmark$ & & & \\
\hline 7 & $1011 \overline{1}$ & $\checkmark$ & & & $\checkmark$ & $\checkmark$ \\
\hline 8 & 10110 & $\checkmark$ & & & v & $\checkmark$ \\
\hline 9 & 10101 & $\checkmark$ & & & v & \\
\hline 10 & 10100 & $\checkmark$ & & & $\checkmark$ & \\
\hline 11 & 1001 & $\checkmark$ & & & & $\checkmark$ \\
\hline 12 & $0110_{-}$ & & $\checkmark$ & & $\checkmark$ & \\
\hline 13 & $0101_{-}$ & & $\checkmark$ & & & $\checkmark$ \\
\hline 14 & $0011_{-}$ & & & & $\checkmark$ & $\checkmark$ \\
\hline
\end{tabular}

${ }^{1}$ Codes for models include a 1/0 digit for each variance component in the following order, direct additive genetic, direct permanent environmental, maternal additive genetic, maternal permanent environmental, and cov if the covariance between direct and maternal additive genetic effects is estimated or set to $0 ; \sigma_{\mathrm{ad}}^{2} / \sigma_{\mathrm{am}}^{2}=$ direct $/$ maternal additive genetic variance; $\sigma_{\mathrm{pd}}^{2} / \sigma_{\mathrm{pm}}^{2}=$ direct $/$ maternal permanent environnemental variance; $\sigma_{\mathrm{adm}}=$ direct-maternal additive genetic covariance. 
Models were solved using the remlf90 package of the blupf90 family of programmes (Misztal et al. 2002). The software provides estimates for the effects of the model as well as for the variance components together with statistics useful for statistical model comparison such as $-2 \log$ likelihood $(2 \log L)$ and the Akaike information criterion (AIC). In order to evaluate the impact of the use of the alternative models in the genetic evaluations and subsequent selection of the best animals, the Pearson correlation between the direct, maternal EBVs predicted from pairs of models for individual kids were calculated in order to evaluate the expected changes in selection of best candidates for reproduction from different models.

\section{Results}

Figure 2 illustrates the change in the weights of local kids as a function of age. This simple presentation of weight shows large variation in individual phenotypes. The dispersion of the weights observed represents a collective expression of the genotypes of kids under arid conditions along 16 years period. Variation in weights at a given age highlights the heterogeneity of this population. Dispersion of weights increases with age probably due to scale effects, but it could also be associated to changes in variability associated with differing environmental effects and their interaction with animal reactivity along the animal's life.

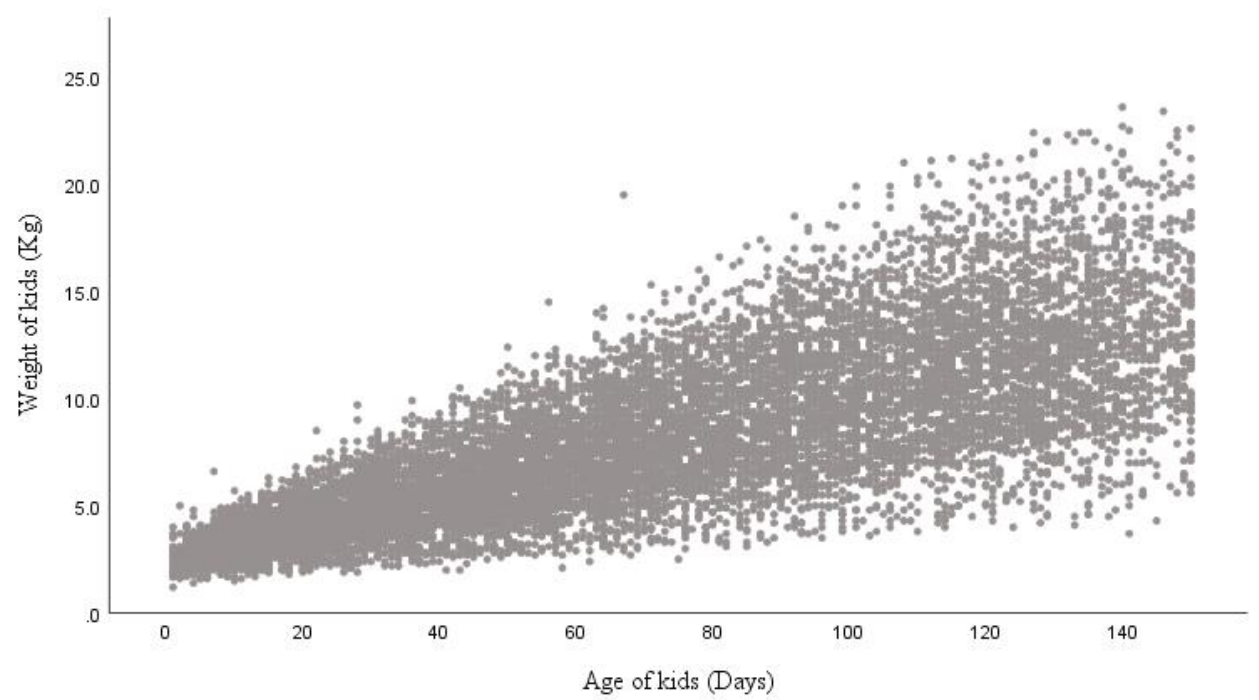

Fig.2 Weight distribution of kids according to their age.

Table 2 provides descriptive statistics of the data used in the study by period of age. Over the four age periods, the number of kids weighed and number of dams of those kids ranged from 552 to 903 and from 217 to 279, respectively. Around 30\% of the dams had records on their own in all age periods. Repeated records were available for kids and dams, 

with less than $10 \%$ of the kids or dams having only one record in P1 and P4 and $10 \%$ of kids/dams having more than $2 / 9$ and 4/17, respectively for those periods. Weights (standard deviations) across periods increased from 3.65(0.96) in P1 to 12.65(3.70) in P4, following the trend presented in Figure2.

Table 2 Statistical description of data by period.

\begin{tabular}{lllll}
\hline & P1 $^{\mathbf{1}}$ & P2 $^{\mathbf{1}}$ & P3 $^{\mathbf{1}}$ & P4 $^{\mathbf{1}}$ \\
\hline No. of records & 911 & 3088 & 4117 & 1415 \\
No. of animals & 691 & 903 & 824 & 552 \\
No. of dams/with own records & $236 / 70$ & $279 / 88$ & $270 / 83$ & $217 / 57$ \\
No. of records/kid-Mean & 1.32 & 3.42 & 4.99 & 2.56 \\
(p10,median,p90) & $(1,1,2)$ & $(2,3,5)$ & $(1,3,8)$ & $(1,2,4)$ \\
No. of records/dam-Mean & 3.86 & 11.07 & 15.25 & 6.52 \\
(p10,median,p90) & $(1,2,9)$ & $(2,7,26)$ & $(2,8,40)$ & $(1,4,17)$ \\
Weights (kg) Mean (SD) & $3.65(0.96)$ & $5.84(1.86)$ & $9.87(3.21)$ & $12.65(3.70)$ \\
\hline
\end{tabular}

${ }^{1}$ Periods defined by age limits, in brackets, $\mathrm{P} 1=[1,20], \mathrm{P} 2=[25,60], \mathrm{P} 3=[65,120], \mathrm{P} 4=[125,150]$.

${ }^{2}(\mathrm{p} 10$,median,p90)=percentile 10,50 and 90 of the distribution of number of records per kid or dam in the data.

Table 3 shows the results of the ANOVA analyses to test the significance of the interaction year $\times$ month of birth and sex $\times$ type of birth, the effect of the age and weight of the dam at kidding on different weights of the kids. Effect of the year-month interaction was significant for all traits. The coefficient of determination $\left(\mathrm{R}^{2}\right)$ increased from 0.60 in $\mathrm{P} 1$ to 0.85 in P4. A relatively high coefficient of determination attests to the importance of these fixed effects included in the adopted model.Effects reflecting environmental and management conditions along time (year-month of kidding) and the effect of sex and type of birth for the kid were highly significant in all periods. Nevertheless, effects relative to groups of dams, i.e., age and weight of dam, showed a small degree of significance overall. As expected, weight of dam at kidding had the largest effect on BW and decreased in relevance as the age of the kid increased. On the other hand, the age of the dam, showed a reverse trend, with largest effect at later ages.

Table 3 Test of significance from ANOVA analyses and coefficient of determination $\left(\mathrm{R}^{2}\right)$ of a model including non-genetic factors on growth traits of local goats by periods of age of kids at recording.

\begin{tabular}{lrrrrr}
\hline Sources of variation & DF & P1 $^{\mathbf{1}}$ & P2 $^{\mathbf{1}}$ & P3 $^{\mathbf{1}}$ & P4 $^{\mathbf{1}}$ \\
\hline Year $\times$ month & 19 & $* *$ & $* *$ & $* *$ & $* *$ \\
\hline Sex $\times$ type of birth & 2 & $* *$ & $* *$ & $* *$ & $* *$ \\
\hline Weight of dam & 2 & $*$ & $*$ & $*$ & NS \\
\hline Age of dam & 7 & $*$ & $*$ & $* *$ & $* *$ \\
\hline $\mathrm{R}^{2}$ & - & 0.60 & 0.67 & 0.78 & 0.85 \\
\hline
\end{tabular}

$\mathrm{DF}=$ degrees of freedom; $*=$ Significant $(\mathrm{p}<0.05) ; * *=$ Significant $(\mathrm{p}<0.01) ; \mathrm{NS}=$ non significant. 
Values for AICs and estimates of variance components and associated genetic parameters are shown in Tables 4 to 7 . For all periods, the best models were those including both DAG and MAG effects. Exclusion of one of these effects resulted in large increases of the AIC. In addition to the two genetic effects, presence or absence of the DPE effect had also a large impact on the goodness of fit of models. On the other hand, absence of the MPE effect resulted in nearly null changes in the AIC. Assuming a null covariance between direct and maternal genetic components did not imply losses in goodness of fit when both,DAG and MAGeffects, were present in the model. Overall, the best model in terms of the AIC valuewas model 4, which includes DAG, DPE and MAG effects assuming a null covariance between DAG ad MAG effects.On the other hand, the worst model was model 14, the one fitting only maternal effects and ignoring direct effects.

Estimates of variance components and genetic parameters are also shown in Tables 4 to 7 for the four periods of age of kids at weighing and Figure 3 shows the ratio of variance components to total variance so that model estimates can be compared across periods.

Table 4 Estimates of (co)variance components and genetic parameter estimates for body weights at first period of age.

\begin{tabular}{|c|c|c|c|c|c|c|c|c|c|c|c|}
\hline \multicolumn{12}{|c|}{ P1 } \\
\hline $\begin{array}{l}\text { Models } \\
\text { Code/No }\end{array}$ & $\sigma_{\mathrm{ad}}^{2}$ & $\sigma_{p d}^{2}$ & $\sigma_{\mathrm{adm}}$ & $\sigma_{\mathrm{am}}^{2}$ & $\sigma_{\mathbf{p m}}^{2}$ & $\sigma_{\mathrm{e}}^{2}$ & $\sigma_{y}^{2}$ & $\mathbf{h}^{2}{ }_{d}$ & $\mathbf{h}^{2} \mathbf{m}$ & $\mathbf{r}_{\mathrm{dm}}$ & AIC \\
\hline $11111 / 1$ & 0.013 & 0.321 & -0.003 & 0.011 & 0.043 & 0.101 & 0.487 & 0.03 & 0.02 & -0.21 & 166 \\
\hline $11110 / 2$ & 0.011 & 0.323 & -- & 0.012 & 0.041 & 0.101 & 0.487 & 0.02 & 0.02 & -- & 164 \\
\hline $11101 / 3$ & 0.013 & 0.328 & -0.004 & 0.051 & -- & 0.101 & 0.489 & 0.03 & 0.10 & -0.15 & 165 \\
\hline $11100 / 4$ & 0.013 & 0.328 & -- & 0.047 & -- & 0.101 & 0.489 & 0.03 & 0.10 & -- & 163 \\
\hline $11010 / 5$ & 0.015 & 0.320 & -- & -- & 0.051 & 0.101 & 0.487 & 0.03 & -- & -- & 1764 \\
\hline 1100_/6 & 0.058 & 0.328 & -- & -- & -- & 0.101 & 0.487 & 0.12 & -- & -- & 1768 \\
\hline 10111/7 & 0.532 & -- & -0.325 & 0.217 & 0.035 & 0.104 & 0.563 & 0.95 & 0.39 & -0.96 & 173 \\
\hline $10110 / 8$ & 0.426 & -- & -- & 0.010 & 0.083 & 0.120 & 0.639 & 0.67 & 0.02 & -- & 213 \\
\hline $10101 / 9$ & 0.540 & -- & -0.336 & 0.257 & -- & 0.104 & 0.565 & 0.96 & 0.45 & -0.90 & 172 \\
\hline $10100 / 10$ & 0.429 & -- & -- & 0.105 & -- & 0.122 & 0.656 & 0.65 & 0.16 & -- & 216 \\
\hline 1001_/11 & 0.426 & -- & -- & -- & 0.091 & 0.120 & 0.637 & 0.67 & -- & -- & 1812 \\
\hline 0110_/12 & -- & 0.336 & -- & 0.051 & -- & 0.101 & 0.488 & -- & 0.11 & -- & 1763 \\
\hline 0101_/13 & -- & 0.330 & -- & -- & 0.055 & 0.101 & 0.486 & -- & -- & -- & 1762 \\
\hline 0011_/14 & -- & -- & -- & 0.021 & 0.085 & 0.378 & 0.484 & -- & 0.04 & -- & 1911 \\
\hline
\end{tabular}

$\sigma_{\mathrm{ad}}^{2}=$ direct additive genetic variance; $\sigma_{\mathrm{pd}}^{2}=$ direct permanent environmental variance; $\sigma_{\mathrm{y}}^{2}=$ phenotypic variance; $\sigma_{\mathrm{pm}}^{2}=$ maternal permanent environmental variance ; $\sigma_{\mathrm{am}}^{2}=$ maternal additive genetic variance; $\sigma_{\mathrm{adm}}=$ direct-maternal additive genetic covariance ; $\sigma_{\mathrm{e}}^{2}=$ residual variance, $\mathrm{h}_{\mathrm{d}}{ }_{\mathrm{d}}=$ direct heritability, $\mathrm{h}^{2}{ }_{\mathrm{m}}=$ maternal heritability; $\mathrm{r}_{\mathrm{dm}}=$ correlation between maternal and direct effects, AIC=Akaike information criterion. 
All variance component estimates increased along periods as a result of a scale effect, but, ratios of variances did not show this trend. Partition of variability in environmental and genetic components showed large differences across models, with similar patterns in all periods. The only component estimates that remained stable across models were the direct permanent environmental variance and the residual variance (except for the models including only direct or only maternal effects). Exclusion of the permanent environmental effects resulted in larger estimates of the corresponding genetic effects (direct and maternal), as could have been expected.

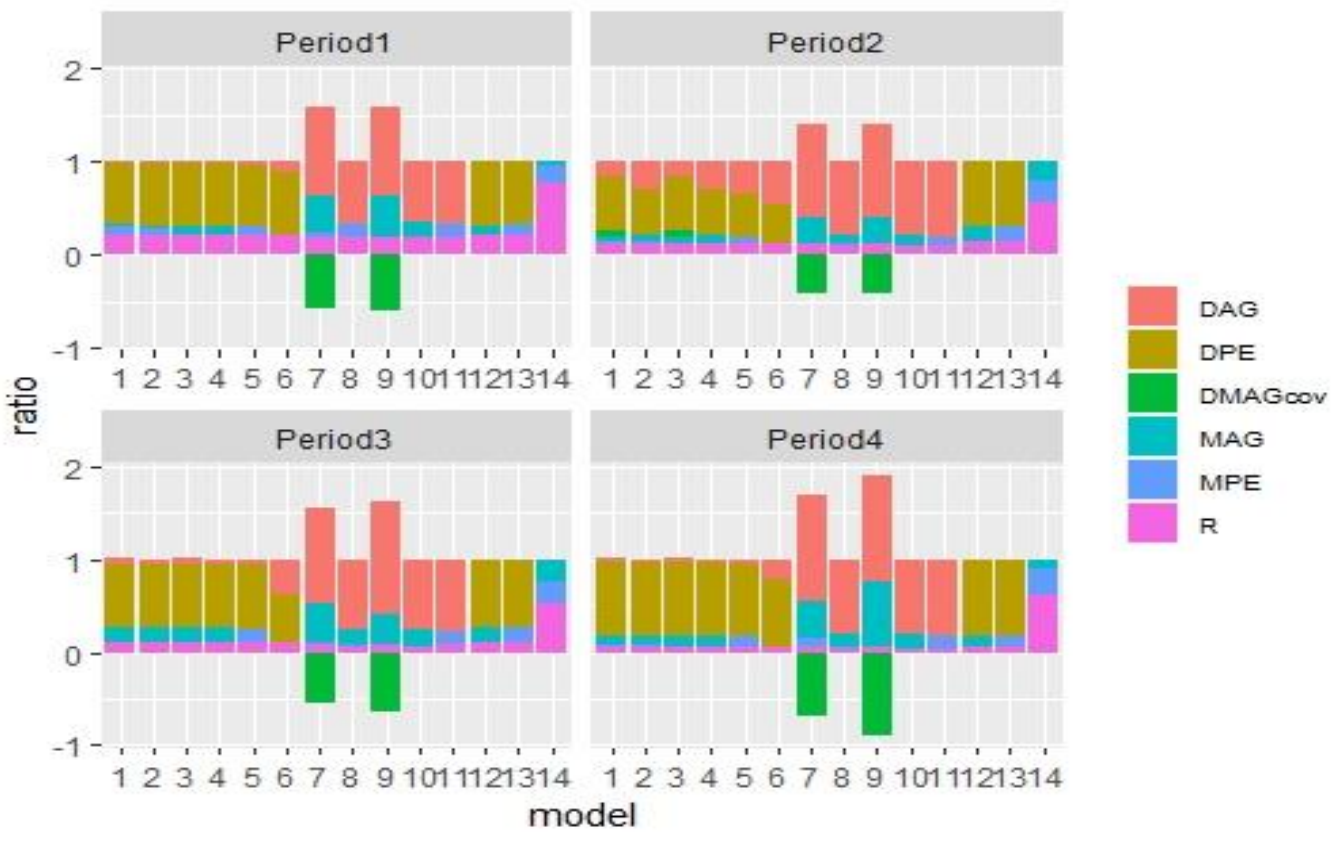

Fig. 3 Ratios of estimated (co)variance components (DAG=Direct Additive Genetic, DPE=Direct Permanent Environmental, DMAGcov= covariance between Direct and Maternal Additive Genetic effects, MAG=Maternal Additive Genetic, MPE $=$ Maternal Permanent Environmental, $\mathrm{R}=$ Residual) to the total variance in the four periods of days of age (Period1 $=[1,20]$, Period $2=[25,60]$, Period $3=[65,120]$, Period $4=[125,150])$ for the fourteen models analysed characterised in Table 1.

Of particular relevance was the impact of exclusion of the DPE effect, which resulted not only in large estimates of the DAG effect but also in the maternal components, and in an apparent overestimation of the total variancefor periods P2, P3 and P4. For P1, the effect on the maternal component was only for the model where a covariance between direct and maternal was fitted. The increase in phenotypic variance in models excluding the direct permanent component was associated with both an increase in the overall direct and also maternal effect and a large negative estimate for the direct-maternal additive genetic covariancecomponent, even for $\mathrm{P} 2$, for which a positive covariance was estimated in other 
models including the direct permanent component. The estimate of the DPE component was between $60 \%$ and $80 \%$ of the total variance in P1 and P4, respectively. Overall, models excluding this component resulted in abnormal estimates of genetic parameters with heritability estimates close to one for the direct and around or over 0.3 for the maternal component, and $r_{d m}$ estimates close to -1 . For other models, heritabilityestimates of both the direct and maternal effects ranged from low $\left(0.02\right.$ for $\mathrm{h}^{2} \mathrm{~m}$ in P1) to moderate $\left(0.17\right.$ for $\mathrm{h}_{\mathrm{d}}^{2}$ and $\mathrm{h}^{2}{ }_{\mathrm{m}}$ in $\mathrm{P} 2$ and P3). Period 1 showed the lowest values for heritability of both direct and maternal effects, which also showed the largest estimate of the ratio of residual to total variance (around 0.2) compared with the other periods, which showed decreasing ratios for the residual variance as age increased (from 0.13 for P2 to 0.07 for P4).

Table 5 Estimates of (co)variance components and genetic parameter estimates for body weights at second period of age.

\begin{tabular}{|c|c|c|c|c|c|c|c|c|c|c|c|}
\hline \multicolumn{12}{|c|}{$\mathbf{P 2}$} \\
\hline $\begin{array}{l}\text { Models } \\
\text { Code/N }\end{array}$ & $\sigma_{a d}^{2}$ & $\sigma_{p d}^{2}$ & $\sigma_{\mathrm{adm}}$ & $\sigma_{\mathrm{am}}^{2}$ & $\sigma_{\mathrm{pm}}^{2}$ & $\sigma_{\mathrm{e}}^{2}$ & $\sigma_{y}^{2}$ & $\mathbf{h}_{\mathrm{d}}^{2}$ & $\mathbf{h}_{\mathrm{m}}^{2}$ & $\mathbf{r}_{\mathrm{dm}}$ & AIC \\
\hline $11111 / 1$ & 0.271 & 0.945 & 0.100 & 0.104 & 0.002 & 0.213 & 1.634 & 0.17 & 0.06 & 0.60 & 4724 \\
\hline $11110 / 2$ & 0.510 & 0.798 & -- & 0.132 & 0.012 & 0.212 & 1.664 & 0.31 & 0.08 & -- & 4722 \\
\hline $11101 / 3$ & 0.274 & 0.943 & 0.100 & 0.106 & -- & 0.213 & 1.635 & 0.17 & 0.06 & 0.59 & 4722 \\
\hline $11100 / 4$ & 0.512 & 0.797 & -- & 0.145 & -- & 0.212 & 1.667 & 0.31 & 0.09 & -- & 4720 \\
\hline $11010 / 5$ & 0.577 & 0.768 & -- & -- & 0.101 & 0.212 & 1.658 & 0.35 & -- & -- & 6761 \\
\hline 1100_/6 & 0.799 & 0.680 & -- & -- & -- & 0.212 & 1.691 & 0.47 & -- & -- & 6761 \\
\hline $10111 / 7$ & 1.871 & -- & -0.765 & 0.532 & 0.019 & 0.213 & 1.870 & 1.00 & 0.28 & -0.77 & 4729 \\
\hline $10110 / 8$ & 1.727 & -- & -- & 0.220 & 0.023 & 0.213 & 2.184 & 0.79 & 0.10 & -- & 4758 \\
\hline $10101 / 9$ & 1.872 & -- & -0.762 & 0.550 & -- & 0.213 & 1.873 & 1.00 & 0.29 & -0.75 & 4727 \\
\hline $10100 / 10$ & 1.728 & -- & -- & 0.249 & -- & 0.213 & 2.190 & 0.79 & 0.11 & -- & 4756 \\
\hline 1001_/11 & 1.730 & -- & -- & -- & 0.198 & 0.213 & 2.141 & 0.81 & -- & -- & 6798 \\
\hline 0110_/12 & -- & 1.113 & -- & 0.279 & -- & 0.213 & 1.604 & -- & 0.17 & -- & 6762 \\
\hline 0101_/13 & -- & 1.113 & -- & -- & 0.259 & 0.213 & 1.585 & -- & -- & -- & 6763 \\
\hline 0011_/14 & -- & -- & -- & 0.351 & 0.369 & 0.955 & 1.675 & -- & 0.21 & -- & 9213 \\
\hline
\end{tabular}


All periods showed similar values of the genetic parameters, with estimated values for $288 \mathrm{~h}^{2}{ }_{\mathrm{m}}$ larger than $\mathrm{h}_{\mathrm{d}}{ }_{\mathrm{d}}$ and close to zero but negative estimates of the $\mathrm{r}_{\mathrm{dm}}$, except $\mathrm{P} 2$, for which $289 \mathrm{~h}_{\mathrm{d}}^{2}$ was larger than $\mathrm{h}^{2} \mathrm{~m}$ with an estimated $\mathrm{r}_{\mathrm{dm}}$ around 0.6.

290 Table 6 Estimates of (co)variance components and genetic parameter estimates for body 291 weights at third period of age.

\begin{tabular}{|c|c|c|c|c|c|c|c|c|c|c|c|}
\hline \multicolumn{12}{|c|}{ P3 } \\
\hline $\begin{array}{l}\text { Models } \\
\text { Code/No }\end{array}$ & $\sigma_{\mathrm{ad}}^{2}$ & $\sigma_{\text {pd }}^{2}$ & $\sigma_{\mathrm{adm}}$ & $\sigma_{a m}^{2}$ & $\sigma_{\mathrm{pm}}^{2}$ & $\sigma_{\mathrm{e}}^{2}$ & $\overline{\sigma_{y}^{2}}$ & $\mathbf{h}^{2}{ }_{d}$ & $\mathbf{h}^{2}{ }_{\mathrm{m}}$ & $\mathbf{r}_{\mathrm{dm}}$ & AIC \\
\hline $11111 / 1$ & 0.33 & 2.76 & .09 & 0.71 & 0.02 & 0.42 & 4.14 & 0.08 & 0.17 & -0.18 & 9224 \\
\hline $11110 / 2$ & 0.25 & 2.81 & -- & 0.64 & 0.02 & 0.42 & 4.14 & 0.06 & 0.16 & -- & 9222 \\
\hline $11101 / 3$ & 0.34 & 2.75 & -0.09 & 0.73 & -- & 0.42 & 4.15 & 0.08 & 0.18 & -0.18 & 9222 \\
\hline $11100 / 4$ & 0.25 & 2.80 & -- & 0.66 & -- & 0.42 & 4.14 & 0.06 & 0.16 & -- & 9220 \\
\hline $11010 / 5$ & 0.25 & 2.83 & -- & -- & 0.58 & 0.42 & 4.08 & 0.06 & -- & -- & 11107 \\
\hline 1100_/6 & 1.62 & 2.22 & -- & -- & -- & 0.42 & 4.26 & 0.38 & -- & -- & 11111 \\
\hline $10111 / 7$ & 4.94 & -- & -2.69 & 2.11 & 0.07 & 0.42 & 4.86 & 1.02 & 0.43 & -0.83 & 9232 \\
\hline $10110 / 8$ & 4.51 & -- & -- & 1.02 & 0.05 & 0.42 & 6.00 & 0.75 & 0.17 & -- & 9274 \\
\hline $10101 / 9$ & 6.02 & -- & -3.11 & 1.61 & -- & 0.42 & 4.93 & 1.22 & 0.33 & -1.00 & 9257 \\
\hline $10100 / 10$ & 4.51 & -- & -- & 1.07 & -- & 0.42 & 6.01 & 0.75 & 0.18 & -- & 9272 \\
\hline 1001_/11 & 4.52 & -- & -- & -- & 0.91 & 0.42 & 5.85 & 0.77 & -- & -- & 11164 \\
\hline 0110_/12 & -- & 2.97 & -- & 0.73 & -- & 0.42 & 4.12 & -- & 0.18 & -- & 11103 \\
\hline 0101_/13 & -- & 2.99 & -- & -- & 0.65 & 0.42 & 4.07 & -- & -- & -- & 11106 \\
\hline 0011_/14 & -- & -- & -- & 1.15 & 1.04 & 2.38 & 4.57 & -- & 0.25 & -- & 15887 \\
\hline
\end{tabular}
$\sigma_{\mathrm{ad}}^{2}=$ direct additive genetic variance; $\sigma_{\mathrm{pd}}^{2}=$ direct permanent environmental variance; $\sigma_{\mathrm{y}}^{2}=$ phenotypic 293 variance; $\sigma_{\mathrm{pm}}^{2}=$ maternal permanent environmental variance; $\sigma_{\mathrm{am}}^{2}=$ maternal additive genetic variance; $294 \sigma_{\mathrm{adm}}=$ direct-maternal additive genetic covariance; $\sigma^{2}=$ residual variance, $\mathrm{h}^{2}{ }_{\mathrm{d}}=$ direct heritability, $295 \mathrm{~h}_{\mathrm{m}}^{2}=$ maternal heritability; $\mathrm{r}_{\mathrm{dm}}=$ correlation between maternal and direct effects, AIC=Akaike 296 information criterion. 
Table 7 Estimates of (co)variance components and genetic parameter estimates for body weights at the fourth period of age.

\begin{tabular}{|c|c|c|c|c|c|c|c|c|c|c|c|}
\hline \multicolumn{12}{|c|}{ P4 } \\
\hline $\begin{array}{l}\text { Models } \\
\text { Code/N }\end{array}$ & $\sigma_{\mathrm{ad}}^{2}$ & $\sigma_{p d}^{2}$ & $\sigma_{\mathrm{adm}}$ & $\sigma_{\mathrm{am}}^{2}$ & $\sigma_{\mathrm{pm}}^{2}$ & $\sigma_{\mathrm{e}}^{2}$ & $\sigma_{y}^{2}$ & $\mathbf{h}^{2}{ }_{d}$ & $\mathbf{h}^{2} \mathbf{m}$ & $\mathbf{r}_{\mathrm{dm}}$ & AIC \\
\hline $11111 / 1$ & 0.32 & 4.26 & -0.09 & 0.58 & 0.07 & 0.37 & 5.52 & 0.06 & 0.11 & -0.20 & 3162 \\
\hline $11110 / 2$ & 0.23 & 4.31 & _ & 0.52 & 0.07 & 0.37 & 5.51 & 0.04 & 0.09 & - & 3160 \\
\hline $11101 / 3$ & 0.35 & 4.25 & -0.11 & 0.66 & - & 0.37 & 5.52 & 0.06 & 0.12 & -0.22 & 3160 \\
\hline $11100 / 4$ & 0.24 & 4.32 & - & 0.58 & - & 0.37 & 5.51 & 0.04 & 0.11 & - & 3158 \\
\hline $11010 / 5$ & 0.32 & 4.27 & - & _- & 0.54 & 0.37 & 5.50 & 0.06 & - & - & 4497 \\
\hline 1100_/6 & 1.21 & 4.01 & - & _- & _- & 0.37 & 5.60 & 0.22 & _- & - & 4499 \\
\hline $10111 / 7$ & 7.58 & - & -4.57 & 2.75 & 0.57 & 0.37 & 6.71 & 1.13 & 0.41 & -1.00 & 3172 \\
\hline $10110 / 8$ & 6.76 & - & _- & 1.18 & 0.07 & 0.37 & 8.39 & 0.81 & 0.14 & - & 3207 \\
\hline $10101 / 9$ & 7.80 & _ & -6.08 & 4.74 & - & 0.37 & 6.83 & 1.14 & 0.69 & -1.00 & 3170 \\
\hline $10100 / 10$ & 6.77 & - & _- & 1.27 & - & 0.37 & 8.41 & 0.81 & 0.15 & _ & 3205 \\
\hline 1001_/11 & 6.79 & - & - & - & 1.05 & 0.37 & 8.20 & 0.83 & - & - & 4547 \\
\hline 0110_/12 & - & $4 . \overline{4}$ & - & 0.62 & - & 0.37 & 5.48 & - & 0.11 & - & 4494 \\
\hline 0101_/13 & - & 4.50 & - & _- & 0.59 & 0.37 & 5.46 & _- & _- & _- & 4496 \\
\hline 0011_/14 & 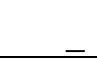 & & - & 0.61 & 1.52 & 3.47 & 5.59 & . & 0.11 & _- & 6017 \\
\hline
\end{tabular}

$\sigma_{\mathrm{ad}}^{2}=$ direct additive genetic variance; $\sigma_{\mathrm{pd}}^{2}=$ direct permanent environmental variance; $\sigma_{\mathrm{y}}^{2}=$ phenotypic variance; $\sigma_{\mathrm{pm}}^{2}=$ maternal permanent environmental variance ; $\sigma_{\mathrm{am}}^{2}=$ maternal additive genetic variance; $\sigma_{\mathrm{adm}}=$ direct-maternal additive genetic covariance ; $\sigma_{\mathrm{e}}^{2}=$ residual variance, $\mathrm{h}_{\mathrm{d}}^{2}=$ direct heritability, $\mathrm{h}^{2} \mathrm{~m}=$ maternal heritability; $\mathrm{r}_{\mathrm{dm}}=$ correlation between maternal and direct effects, AIC=Akaike information criterion.

Correlations between EBVs for direct and maternal effects across models are shown in Tables 8 to 11 for models sharing direct or maternal effects. Accordingly, with what was observed for the variance components estimates, fitting direct permanent effects as well as direct genetic effects had a large impact on the EBVs for both the direct (DEBVs) and maternal (MEBVs) effects. Both direct and maternal EBVs showed high correlations between models fitting DAG and DPE effects. For DEBVs, correlations were above 0.99, indicating that the same animals are expected to be selected under any model that includes direct genetic and direct permanent effects, regardless of the maternal effects included. For MEBVs, correlations were also high, but slightly lower than for the DEBVs between models including DGP effects and maternal genetic effects. EBVs correlation for models including DAG and DPE effects (Models 1 to 6) with and without covariance between direct and maternal effects remained high, even for $\mathrm{P} 2$, where the value of the covariance was substantial. 
321 Table 8 Pearson correlations between estimated breeding values between models for direct 322 (above diagonal) and maternal (below diagonal) effects on body weights in Period 1.

\begin{tabular}{lrrrrrrrrrrr}
\hline \multicolumn{10}{c}{ P1 } \\
\hline Models/No & $\mathbf{1}$ & $\mathbf{2}$ & $\mathbf{3}$ & $\mathbf{4}$ & $\mathbf{5}$ & $\mathbf{6}$ & $\mathbf{7}$ & $\mathbf{8}$ & $\mathbf{9}$ & $\mathbf{1 0}$ & $\mathbf{1 1}$ \\
\hline $11111 / 1$ & 1 & 0.99 & 0.99 & 0.99 & 0.99 & 0.88 & 0.59 & 0.7 & 0.59 & 0.68 & 0.7 \\
$11110 / 2$ & 0.98 & 1 & 0.98 & 1 & 1 & 0.91 & 0.53 & 0.68 & 0.53 & 0.66 & 0.69 \\
$11101 / 3$ & 0.99 & 0.99 & 1 & 0.98 & 0.97 & 0.84 & 0.63 & 0.69 & 0.63 & 0.68 & 0.69 \\
$11100 / 4$ & 0.98 & 0.99 & 1 & 1 & 1 & 0.92 & 0.56 & 0.71 & 0.55 & 0.69 & 0.72 \\
$11010 / 5$ & - & - & - & - & 1 & 0.92 & 0.54 & 0.7 & 0.53 & 0.68 & 0.71 \\
$1100 \_/ 6$ & - & - & - & - & - & 1 & 0.55 & 0.79 & 0.54 & 0.77 & 0.8 \\
$10111 / 7$ & 0.19 & 0.05 & 0.08 & 0.05 & - & - & 1 & 0.86 & 1 & 0.88 & 0.86 \\
$10110 / 8$ & 0.91 & 0.91 & 0.9 & 0.9 & - & - & 0.16 & 1 & 0.85 & 0.99 & 1 \\
$10101 / 9$ & 0.35 & 0.22 & 0.25 & 0.22 & - & - & 0.98 & 0.31 & 1 & 0.87 & 0.85 \\
$10100 / 10$ & 0.89 & 0.88 & 0.9 & 0.9 & - & - & 0.17 & 0.98 & 0.32 & 1 & 0.99 \\
$1001 \_/ 11$ & - & - & - & - & - & - & - & - & - & - & 1 \\
\hline
\end{tabular}

323

324

325

Table 9 Pearson correlations between estimated breeding values between models for direct (above diagonal) and maternal (below diagonal) effects on body weights in Period 2.

\begin{tabular}{lrrrrrrrrrrr}
\hline \multicolumn{10}{c}{ P2 } \\
\hline Models/No & $\mathbf{1}$ & $\mathbf{2}$ & $\mathbf{3}$ & $\mathbf{4}$ & $\mathbf{5}$ & $\mathbf{6}$ & $\mathbf{7}$ & $\mathbf{8}$ & $\mathbf{9}$ & $\mathbf{1 0}$ & $\mathbf{1 1}$ \\
\hline $11111 / 1$ & 1 & 0.97 & 1 & 0.97 & 0.98 & 0.97 & 0.78 & 0.87 & 0.78 & 0.86 & 0.88 \\
$11110 / 2$ & 0.9 & 1 & 0.97 & 1 & 1 & 0.99 & 0.89 & 0.94 & 0.89 & 0.94 & 0.95 \\
$11101 / 3$ & 1 & 0.91 & 1 & 0.97 & 0.98 & 0.98 & 0.78 & 0.87 & 0.78 & 0.87 & 0.88 \\
$11100 / 4$ & 0.9 & 1 & 0.91 & 1 & 1 & 0.99 & 0.89 & 0.94 & 0.89 & 0.94 & 0.95 \\
$11010 / 5$ & - & - & - & - & 1 & 0.99 & 0.88 & 0.94 & 0.88 & 0.94 & 0.95 \\
$1100 \_/ 6$ & - & - & - & - & - & 1 & 0.86 & 0.93 & 0.86 & 0.93 & 0.94 \\
$10111 / 7$ & -0.22 & 0.18 & -0.22 & 0.18 & - & - & 1 & 0.96 & 1 & 0.97 & 0.95 \\
$10110 / 8$ & 0.61 & 0.83 & 0.62 & 0.83 & - & - & 0.39 & 1 & 0.97 & 1 & 1 \\
$10101 / 9$ & -0.19 & 0.22 & -0.18 & 0.21 & - & - & 1 & 0.42 & 1 & 0.97 & 0.95 \\
$10100 / 10$ & 0.61 & 0.83 & 0.62 & 0.83 & - & - & 0.39 & 1 & 0.42 & 1 & 1 \\
$1001 \_/ 11$ & - & - & - & - & - & - & - & - & - & - & 1 \\
\hline
\end{tabular}

326

327

328

329

330

331

332

333

334

Correlations for EBVs from models where direct permanenteffects were ignored (Models 7 to 11) weremuch lower. This was especiallytrue for correlations between MEBVs undemodels with non-nullgenetic maternal effects, which were near 0 or slightly negative because of the high negative value estimates of the genetic correlation between direct and maternal additive effects. 
339

\section{0}

Table 10 Pearson correlations between estimated breeding values between models for direct (above diagonal) and maternal (below diagonal) effects on body weights in Period 3.

\begin{tabular}{lrrrrrrrrrrr}
\hline \multicolumn{10}{c}{ P3 } \\
\hline Models/No & $\mathbf{1}$ & $\mathbf{2}$ & $\mathbf{3}$ & $\mathbf{4}$ & $\mathbf{5}$ & $\mathbf{6}$ & $\mathbf{7}$ & $\mathbf{8}$ & $\mathbf{9}$ & $\mathbf{1 0}$ & $\mathbf{1 1}$ \\
\hline $11111 / 1$ & 1 & 0.98 & 1 & 0.98 & 0.97 & 0.82 & 0.8 & 0.82 & 0.8 & 0.82 & 0.81 \\
$11110 / 2$ & 1 & 1 & 0.98 & 1 & 0.99 & 0.87 & 0.73 & 0.8 & 0.75 & 0.8 & 0.8 \\
$11101 / 3$ & 1 & 0.99 & 1 & 0.98 & 0.97 & 0.82 & 0.8 & 0.82 & 0.8 & 0.82 & 0.82 \\
$11100 / 4$ & 1 & 1 & 1 & 1 & 0.99 & 0.87 & 0.73 & 0.8 & 0.76 & 0.8 & 0.8 \\
$11010 / 5$ & - & - & - & - & 1 & 0.86 & 0.69 & 0.76 & 0.72 & 0.76 & 0.77 \\
$1100 \_/ 6$ & - & - & - & - & - & 1 & 0.73 & 0.87 & 0.81 & 0.87 & 0.89 \\
$10111 / 7$ & 0.32 & 0.24 & 0.33 & 0.24 & - & - & 1 & 0.93 & 0.97 & 0.93 & 0.92 \\
$10110 / 8$ & 0.79 & 0.76 & 0.79 & 0.77 & - & - & 0.44 & 1 & 0.93 & 1 & 1 \\
$10101 / 9$ & -0.19 & -0.27 & -0.19 & -0.27 & - & - & 0.84 & 0.05 & 1 & 0.93 & 0.92 \\
$10100 / 10$ & 0.79 & 0.76 & 0.79 & 0.77 & - & - & 0.44 & 1 & 0.05 & 1 & 0.99 \\
$1001 \_/ 11$ & - & - & - & - & - & - & - & - & - & - & 1 \\
\hline
\end{tabular}

Table 11 Pearson correlations between estimated breeding values between models for direct (above diagonal) and maternal (below diagonal) effects on body weights in Period 4.

\begin{tabular}{lrrrrrrrrrrr}
\hline \multicolumn{10}{c}{} & \multicolumn{10}{c}{$\mathbf{P 4}$} \\
\hline Models/No & $\mathbf{1}$ & $\mathbf{2}$ & $\mathbf{3}$ & $\mathbf{4}$ & $\mathbf{5}$ & $\mathbf{6}$ & $\mathbf{7}$ & $\mathbf{8}$ & $\mathbf{9}$ & $\mathbf{1 0}$ & $\mathbf{1 1}$ \\
\hline $11111 / 1$ & 1 & 0.98 & 1 & 0.98 & 0.96 & 0.86 & 0.74 & 0.8 & 0.63 & 0.8 & 0.81 \\
$11110 / 2$ & 0.99 & 1 & 0.97 & 1 & 1 & 0.91 & 0.63 & 0.77 & 0.49 & 0.76 & 0.78 \\
$11101 / 3$ & 1 & 0.99 & 1 & 0.97 & 0.95 & 0.85 & 0.76 & 0.81 & 0.65 & 0.81 & 0.81 \\
$11100 / 4$ & 0.99 & 1 & 0.99 & 1 & 1 & 0.91 & 0.64 & 0.77 & 0.49 & 0.77 & 0.78 \\
$11010 / 5$ & - & - & - & - & 1 & 0.91 & 0.61 & 0.75 & 0.46 & 0.75 & 0.77 \\
$1100 \_/ 6$ & - & - & - & - & - & 1 & 0.62 & 0.83 & 0.42 & 0.83 & 0.85 \\
$10111 / 7$ & 0.01 & -0.08 & 0.02 & -0.08 & - & - & 1 & 0.87 & 0.96 & 0.87 & 0.86 \\
$10110 / 8$ & 0.74 & 0.71 & 0.76 & 0.72 & - & - & 0.2 & 1 & 0.73 & 1 & 1 \\
$10101 / 9$ & 0.2 & 0.12 & 0.22 & 0.12 & - & - & 0.96 & 0.34 & 1 & 0.73 & 0.71 \\
$10100 / 10$ & 0.74 & 0.71 & 0.75 & 0.72 & - & - & 0.2 & 1 & 0.34 & 1 & 1 \\
$1001 \_/ 11$ & - & - & - & - & - & - & - & - & - & - & 1 \\
\hline
\end{tabular}

\section{Discussion}

Kids of this local breed were characterized by low weights. Similar results have been reported in most breeds and local populations raised under arid environments (Heydarpour et al. 2008; Al-Shorepy et al. 2002). Such a low weight of local kids corresponds to low energetic needs, which could be considered as epigenetic strategy of adaptation to the difficult environmental conditions. In this regard, low weights can be regarded are indeed specific mechanisms of adaptation to the restrictive and irregular environment as shown by (Najari 2005, Paola et al. 2011). The adaptation mechanism could be either genetic or epigenetic as these harsh and limiting environments may result in epigenetic marks oriented to limit the size of animals to endure starvation and heat-stress, as it has been observed in some African human population (Zhang et al. 2008). 
Systematic environmental effects representing changes in the production environment, including age and weight of dams, and effects linked to the kid were highly significant across all ages. The effect of the year-month interaction captures variations of climate, food nutritional quality and herd management along time, which change greatly in extensive conditions. As for the effect of sex and type of birth of the kid, it is a well-known fact that females show lower weights than males and singletons larger weights that kid from multiple births. Present findings were in good agreement with the findings of (Mavrogenis et al. 1984; Maniatis and Pollott 2003; Portolano et al. 2002; Přibyl et al. 2008). On the other hand, systematic effects describing the effect of dam's characteristics (weight and age) showed smaller significance in some periods, with the unexpected result that age of dam had a more significant effect at later ages of the kid, while weight of the dam showed a decreasing significance from birth to the post-weaning period (P4), for which it did not show significance. In growing animals, weight and age are correlated, thus, for young damsin their first kidding, that correlation may affect the significance of both, weight and age effects. Regardless of the collinearity,weight and age are included in the model because they attempt to correct for different biological effects; on one hand weight accounts for the body condition of the dam to face the lactation and age of the dam accounts for the changing physiology of milk production of the dams according to age.

As in previous studies (Mugambi et al. 2007; Misztal et al. 2002; Rashidi et al. 2015; Szwaczkowski et al. 2006), the magnitude and biological meaning of variance and covariance components changed substantially across the complete and reduced models. Nevertheless, for any model, even when comparing a model fitting only direct (model 10) or only maternal components (model 14), direct effects absorbed a substantially larger portion of the total variation than maternal effects. Residual variance was substantially larger in model 14 (range of the residual to total variance ratio ranged from 0.52 for $\mathrm{P} 3$ to 0.78 for P1), which fits only maternal effects, while model 6, which fits only direct effects, showed the same residual variance as the complete model in all periods. Although a smaller source of variation, maternal effects had a substantial contribution to the observed variability, which remained relevant even for the last period, when kids have already been weaned. The MEBV at weaning estimates the milking ability of the dam during the pre-weaning period (David et al. 2015). Since both direct and maternal components are expressed in the same individual, the GxE interaction relationship between the genotype of the kid and the environmental conditions provided by its dam, which could occur during gestation or early in life, may influence individual performance all along their productive life. This environmental impact of 
the dam on its offspring could be captured by a maternal component on weights after weaning as has been estimated in other species such as in beef cattle populations for yearly even $18^{\text {th }}$ month weights (Meyer 1992b; Ngere et al. 2017; Robinson et al. 1996) meat sheep breeds (Heydarpour et al. 2008), as well as goat breeds (Gunia et al. 2011) that has been always interpreted as a carry-over effect from weaning. Though in a restricted environment as the one we have here it could just indicate a competing effect between dam and offspring for food resources since P4 is the dry period with more restricted feeding.

In general, as expected, there was a clear dependency between permanent environmental and additive genetic components for both direct and maternal effects, since both are linked to the same individual. When one of the two components was removed the other one absorbed the remained variability. Disentangling components of variance in complex models, such as the direct and maternal effects models in the analysis of weights, may be subject to identifiability problems. Several authors have dealt with identifiability of parameters in animal breeding models (Bahreini et al. 2007; David et al. 2015; Meyer 1992b) finding that distribution of kinship information and data structure plays a role in the identifiability of the (co)variance components. In our study, direct permanent was by far the largest component of variance (between 60 and $80 \%$ of the total variability depending on the age period), with a likely underestimation of the additive genetic effects and smaller than expected estimates of heritability for both direct and maternal components. In order to further investigate this confounding between direct and maternal effects, Pearson correlations between these two components of direct and maternal effects were obtained. A large correlation between those two effects was found in our data for all models that fitted both effects simultaneously. For direct effects, the Pearson correlation between additive genetic and permanent environmental effects for the same animal ranged from 0.53 for P1 under the full model without covariance (model 2) to 0.83 for P2 under the model with only direct effects (model 6). For maternal effects, correlations were higher, with values above 0.85 for all models.

Proper separation of additive genetic and permanent environmental effects requires the existence of repeated records for kids and dams and complete pedigrees. In this study, repeated weight records were available for each kid and each dam, with P1 having the smallest degree of repeated records for kids (1.3 records/kid on average) and dams (3.9 records of progeny/dam). In fact, this could be the explanation for the much smaller ratio of direct additive genetic to direct permanent environmental effects (4.5\%) in P1 compared with the rest of the periods $(29,12$ and $8 \%$ for P2, P3 and P4, respectively). In the same manner, maternal additive genetic was a $22 \%$ of the maternal permanent environmental variability in 
P1 while in the rest of the periods maternal additive genetic was nearly $100 \%$ of the total maternal variability. The lack of relevance of maternal permanent environmental effect might also be associated with the adjustment of weight and age of dam systematic effects, which may be removing part of the dam's environmental effects contributing to the kid's weight.

Several studies have also pointed at the importance of complete datasets with more links between mother performance records and offspring records, as well as the presence of specific type of family relationships to be able, to obtain reliable estimates of maternal effects (Meyer 1992b; Maniatis et al. 2003; Lee et al. 2000). Thus, (Meyer, 1992b) in a simulation study compared several family structures propose by a number of authors to separate direct and maternal components as well as genetic from permanent environmental components. She pointed out the need to of specific family structures that permit the separation of those effects. In our case, given the large period of years analysed, there were a number of goats that were dams or grand dams of kids with records: around $30 \%$ of the dams of kids with data had records on their own (57 to 88 goats across periods), $5 \%$ (9 to 15 goats across periods) were maternal grand dams of kids with records on their own and one goat was great maternal grandmother and had own record. Thus, the link between performance of mothers and kids was present in this data to a certain extent. This together with a reasonably large number of progeny records per dam may suffice to obtain reliable estimates of maternal effects.

Period 2 was the period showing a distinct pattern of genetic parameter estimates, with the largest proportion of direct additive genetic to total variance and a substantially positive correlation (0.6) between direct and maternal additive genetic effects while this correlation showed close to null or slightly negative values in other periods. Period 2, covering the range of 25 to 60 days of age, is characterised by containing the peak of milk production which implies that kids are likely to be able to cover the growth needs from milk and not depend on availability of pasture or concentrate feeding. Under these conditions, full expression of the growing potential might be expected, which, together with a large number of repeated records, might explain the larger variability in additive genetic values estimated in this period.

The positive correlation estimated between direct and maternal additive genetic effects implies that goats that grow more when kids are better mothers (produce more milk) during P2. A positive correlation between both the aptitude to grow and to be a better mother would make sense in a harsh environment like the one in this study in the context of robust or resilient animals that show also better productive ability for milk and growth along their lives. Showing the potential for production, either as a growing animal or as a lactating dam, likely require favourable environmental conditions that may not be present along the productive 
cycle. Period 2 seems to be the most favourable period for both growing of kids because of favourable milk production of dams and for dams since this period coincides with favourable environmental conditions in terms of pasture and ambient temperatures in the months of January and February. Although the most common result for the genetic correlation between direct and maternal genetic component, positive values have also been found by (Heydarpour, et al. 2008; Al-Shorepy et al. 2002; Diwu et al. 2010; Ekiz et al. 2005) for local breeds of goats from arid areas.

Overall, despite of possible confounding between additive genetic and permanent environmental effects, no serious consequences for selection decisions are expected from using any of the models containing DAG, DPE and MAG effects, regardless of the assumption about the covariance between additive genetic effects. [David et al 2015] had observed a similar result when analysing weights from three very different livestock species (pigs, sheep and rabbits).These high correlations between EBVs regardless the model, opens a windows of opportunity to establish a genetic evaluation program in this experimental farm, with the goal of been a genetic resource for farmers of the breed.

\section{Conclusion}

Both direct and maternal effects were relevant sources of variation in all periods but direct effects represented a much larger proportion of the total variability observed. The direct permanent environmental effect was particularly strong while the maternal permanent environmental effect was nearly irrelevant. Overall, models including both direct and maternal additive genetic and the direct permanent environmental effects were best in terms of goodness of fit and in terms of separation of components of variance. Assuming a null correlation between direct and maternal additive genetic effects did not improve the goodness of fit and had nearly no impact on the genetic evaluations of both direct and maternal effects and consequently on selection decisions. Identification of genetic and environmental components of weight variability under maternal and direct effects require substantial amount of repeated records and genetic links between animals and it should be further pursued to determine the optimal design of recording for genetic evaluations. Along all periods of age of the kid, we conclude that periods that allow the full expression of the genetic potential for growth and for milk production of the dam (P2, comprising day 25 to 60 of age, in our study) would be the optimal time for recording in this type of production system. 
487 Funding: The authors declare they have no funding.

488 Conflict of interest: The authors declare that they have no conflict of interest.

489 Ethics approval: This study does not involve any human or animal testing, only routine

490

491

492

493

494

495

496

497

498

499

500

501

502

503

504

505

506

507

508

509

510

511

512

513

514

515

516

517

518

519 management practices in animal husbandry.

\section{Consent to participate: na}

Consent for publication: na

Availability of data and material : The data used in this study are available on request from the corresponding author.

\section{Code availability:na}

Author Contributions: Conceptualization, methodology and validation, A.A., M.J.C., S.N. and C.D.; investigation and data collection and analysis, A.A., M.J.C., S.N. and M.A.; writing - original draft preparation, A.A. and M.J.C.; writing - review and editing, A.A., M.J.C., S.N. and C.D.; supervision and project administration, M.J.C. and S.N.; All authors have read and agreed to the published version of the manuscript.

\section{References}

Al-Shorepy, S.A., Alhadranu, G.A. and Abdul Wahab, K., 2002. Genetic and phenotypic parameters for early growth traits in Emirati goat. Small Ruminant Research, 45,217223.

Atoui, A., Carabaño, M.J., Diaz, C. and Najari, S., 2019. Genetic analysis of live weight of local kids to promote genetic evaluations in the arid areas of Tunisia. Tropical Animal Health Production, 1, 1-14.

Atoui, A., Carabaño, M.J., Abdenebi, M.and Najari, S., 2018. Evaluation of a local goat population for fertility traits aiming at the improvement of its economic sustainability through genetic selection. Spanish Journal of Agriculture Research, 16, 404-411.

Barazandeh, A.,2006. Maternal effects on pre- and post-weaning growth traits in Kermani sheep. Animal Science. TarbiatmodaresUniversity, Tehran. p70.

Bahreini, M.R., Shahroudi, F.E.and Van Vleck, L.D., 2007. Estimates of genetic parameters for growth traits in Kermani sheep. Journal of Animal Breeding,124, 296-301.

Bijma, P.,2006. Estimating maternal effects in livestock. Journal of Animals Sciences, 84, 800-806.

Boujenane, I. and Kansari, J., 2002. Estimates of (co)variances due to direct and maternal effects for body weights in Timahdite sheep. Animal Science, 74, 409-414. 
David, I., Bouvier, F., Banville, M., Canario, L., Flatres-Grall, L., Balmisse, E.and Garreau, H.,2015. The direct-maternal genetic correlation has little impact on genetic evaluations. Journal of Animal Science, 93, 5639-5647.

DiWu, El., François, V., Marie, L., Jane, E., Visvader, G. and Smyth, K., 2010. Rotation gene set tests for complex micro array experiments. Bioinformatics, 26, 2176-2182.

Ekiz, B., 2005. Estimates of maternal effects for pre-and postweaning daily gain in Turkish merino lambs. Turkish Journal of Veterinary Animal Science, 29, 399- 407.

Fischer, T., Vanderwert, M., Banks, J.H.J. and Ball, G.AJ., 2004. Description of lamb growth using random regression on field data. Livestock Production Science, 89, 175-185.

Ghafouri Kesbi, F., Eskandarinasab, M. and Hassanabadi, A.,2008. Estimation of genetic parameters for lamb weight at various ages in Mehraban sheep. Italian Journal of Animal Science, 7, 95-103.

Gunia,M., Phocas, F., Arquet,R., Alexandre,G. and Mandonnet, N., 2011. Genetic parameters for body weight, reproduction, and parasite resistance traits in the Creole goat. Journal of Animal Science,89, 3443-3451.

Heydarpour, M., Schaefferand, L.R. and Yazdi, M.H., 2008. Influence of population structure on estimates of direct and maternal parameters. Journal of Animal Breeding ,125, 89-99.

Krejčová, H., Přibyl, J., Přibylová, J., Štipková, M. and Mielenz, N., 2008. Genetic evaluation of daily gains of dualpurpose bulls using random regression model. Journal of Animal Science, 53, 227-237.

Lee, J. W., Choi, S.B., Jung,Y.H., Keown, J. and Van Vleck, L.D., 2000. Parameter estimates for direct and maternal genetic effects on yearling, eighteen-month, and slaughter weights of Korean native cattle. Journal of Animal Science,78, 1414-1421.

Mandal, A., Neser, F.W.C., Rout, P.K., Roy, R.and Notter, D.R., 2006. Genetic parameters for direct and maternal effects on body weights of Muzaffarnagari sheep. Journal of Animal Science, 82, 133-140.

Maniatis, N. and Pollott, G.E., 2003. The impact of data structure on genetic(co)variance components of early growth in sheep, estimated using an animal model with maternal effects. Journal of Animal Science, 81, 101-108. 
Mavrogenis, A.P., Constantinou, A. and Louca, A., 1984. Environmental and genetic causes of variation in production traits of Damascus goats: Pre-weaning and post-weaning growth. Animal Production, 38, 91-98.

Meyer. K., 1992b. Variance components due to direct and maternal effects for growth traits of Australian beef cattle. Livestock Production Science,31, 179-204

Meyer,K.,1992a. Bias and sampling covariances of estimates of variance components due to maternal effects. Genetic Selection Evolution, 30, 24-28.

Mugambi, J.N., Wakhungu, J.W., Inyangala, B.O., Muhuyi, W.B. and Muasya, T.,2007. Evaluation of the performance of the Kenya dual purpose goat composites: additive and non-additive genetic parameters. Small Ruminant Research 72, 149-156.

Misztal, I., Tsuruta, S., Strabel, T., Auvray, B., Druet, T. and Lee, D.H., 2002. BLUPF 90 and related programs (BGF90). In: 7th WCGALP; 19-23, Montpellier, France. 28-07.

Najari, S., 2005. Caractérisation zootechnique et génétique d'une population caprine. Cas de la population caprine locale des régions arides tunisiennes. Thèse de doctorat d'Etat. Institut National AgronomiqueTunisie. p 214.

Ngere, L., Burke, J.M., Notter, D.R. and Morgan, J.L.M., 2017. Variance components for direct and maternal effects on body weights of Katahdin lambs. Journal of Animal Science, 95, 3396-3405.

Paola, T., Cristina, M., Sara, P., Giada, B. and Mauro, B., 2011. Secular trends in growth of African Pygmies and Bantu. Hormones, 10, 144-148.

Portolano, B., Todaro, M., Finocchiaro, R. and van Kaam, J.H.B.C.M., 2002. Estimation of genetic and phenotypic variance of several growth traits of the Sicilian Girgentana goat. Small Ruminant Research, 45, 247-253.

Přibyl, J., Krejčová, H., Přibylová, J., Misztal, I., Tsuruta, S. and Mielenz, N., 2008. Models for evaluation of growth of performance tested bulls. Czech Journal of Animal Science, $53,45-54$.

Rashidi, A., Mokhtari, M.S. and Gutiérrez, J.P., 2015. Pedigree analysis and inbreeding effects on early growth traits and greasy fleece weight in Markhoz goat. Small Ruminant Research, 124, 1-8.

Robinson, D.L., 1996. Estimation and interpretation of direct and maternal genetic parameters for weights of Australian Angus cattle. Livestock Production Science, 46,129-130. 
Szwaczkowski, T., Wojtowski, J., Stanislawska, E. and Gut, A., 2006. Estimates of maternal genetic and permanent environmental effects in sheep. Journal of Animal Science, 49,186-192.

Varona, L., Munilla, S., Casellas, J. and Altarriba, J., 2015. Consequences of paternally inherited effects on the genetic evaluation of maternal effects. Genetic Selection Evolution,50, 47-63.

Zhang, C., Yang, L. and Shen, Z., 2008. Variance components and genetic parameters for weight and size at birth in the Boer goat. Livestock Production Science, 115, 73-79. 
